\title{
Effects of sub-Tenon's capsule triamcinolone acetonide injection combined with microa- neurysm photocoagulation on diabetic macular edema
}

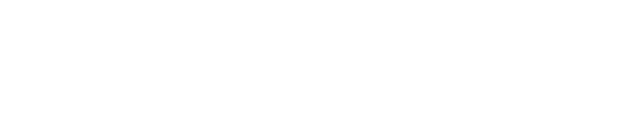

\section{Toshiyuki Oshitari \\ Sakiko Nonomura \\ Miyuki Arai \\ Yoko Takatsuna \\ Eiju Sato \\ Takayuki Baba \\ Shuichi Yamamoto}

Department of Ophthalmology and Visual Science, Graduate School of Medicine, Chiba University, Chuo-ku, Chiba, Japan
Correspondence: Toshiyuki Oshitari Department of Ophthalmology and Visual Science, Graduate School of Medicine, Chiba University, Inohana I-8-I, Chuo-ku, Chiba 260 8670, Japan Tel +8I 432262124 Fax +8I 432244162

Email tarii@aol.com; oshitari@faculty. chiba-u.jp
Purpose: To compare the effects of sub-Tenon's capsule triamcinolone acetonide (STTA) injection to that of STTA injection combined with microaneurysm photocoagulation (MAPC; STTA + MAPC) on eyes with diabetic macular edema (DME).

Patients and methods: The medical records of 138 eyes of 138 patients with DME treated by either STTA or STTA + MAPC were reviewed. The degree of DME was determined by the optical coherence tomographic features: patients with serous retinal detachment (SRD+; 38 eyes) and patients without SRD (non-SRD; 100 eyes). The central macular thickness (CMT) and the best-corrected visual acuity (BCVA) were measured periodically for 6 months after the treatments.

Results: The BCVA was significantly improved in the non-SRD group after STTA + MAPC. The CMT was significantly improved in all groups and improved considerably more in the nonSRD group than in the SRD+ group after STTA + MAPC.

Conclusion: Our findings indicate that MAPC has an additive effect in the non-SRD type.

Keywords: diabetic macular edema, sub-Tenon's triamcinolone acetonide injection, microaneurysm photocoagulation, best-corrected visual acuity, central macular thickness

\section{Introduction}

Even though diabetic macular edema (DME) is a major cause of moderate vision reduction in patients with diabetic retinopathy, the best treatment for DME is still undetermined. ${ }^{1}$ The classical treatment for DME established by the ETDRS (early treatment diabetic retinopathy study) is focal/grid laser photocoagulation. ${ }^{2}$ However, many adverse effects have been reported after laser photocoagulation, eg, retinal fibrosis, atrophic creep, foveal burn, and central visual field defects. ${ }^{3}$

Several other therapies have been used for DME such as corticosteroids, ${ }^{4-6}$ antivascular endothelial growth factor (VEGF) antibodies, ${ }^{7-10}$ and subthreshold micropulse diode laser photocoagulation. ${ }^{11}$ However, a single treatment by these therapies was not sufficient for improving the DME for a long period in some cases. Thus, combination therapies such as anti-VEGF agents combined with laser photocoagulation or intravitreal corticosteroid injection combined with laser photocoagulation have been used to treat refractory DME. ${ }^{12}$

Recent studies have shown that high rates of microaneurysm (MA) formation and high MA turnover rates are significant risk factors for developing and progression of clinically significant macular edema. ${ }^{13,14}$ The MAs can be the blood-retinal barrier 
breakdown conduit, leading to an increase of vascular permeability. Thus, MA photocoagulation (MAPC) is one of the therapies for DME. ${ }^{15}$

Although the precise mechanism that is involved in the development of DME is still undetermined, the results of several studies have indicated that intraocular inflammation induced by diabetic stress is related to the development of DME. ${ }^{16}$ Steroids are anti-inflammatory regents, and they are known to reduce the expression of $\mathrm{VEGF}^{17}$ and upregulate tight junction proteins in endothelial cells. These changes lead to a tightening of the retinal-blood barrier. ${ }^{18}$ Thus, a subTenon's capsule triamcinolone acetonide (STTA) injection or an intravitreal triamcinolone acetonide (IVTA) injection has been used to treat DME. ${ }^{4-6}$ Because IVTA has a higher risk of endophthalmitis and elevation of the intraocular pressure than STTA, ${ }^{19}$ STTA is routinely used to treat DME. ${ }^{5}$ However, a search of PubMed did not extract any publication reporting the additive effects of STTA and MAPC in patients with DME.

Optical coherence tomography (OCT) enables clinicians to identify the morphological differences in eyes with DME, such as those with cystoid macular edema and serous retinal detachment (SRD). ${ }^{20}$ Some investigators have evaluated the effects of IVTA on the SRD in eyes with DME. ${ }^{21-24}$ However, the results of these evaluations were inconsistent; some studies indicated that IVTA was effective for SRD in eyes with DME, ${ }^{21,22}$ but others reported that IVTA was effective for only the cystoid macular edema (CME) type of eyes with DME. ${ }^{23,24}$

Thus, the purpose of this study is to evaluate the effect of STTA on SRD in eyes with DME and also to evaluate the additive effects of STTA combined with MAPC on DME.

\section{Patients and methods}

The medical records of 138 eyes of 138 patients with DME treated with STTA or with STTA + MAPC between January 2010 and July 2011 at the Chiba University Hospital were reviewed. All of the procedures conformed to the tenets of the World Medical Association Declaration of Helsinki. A signed informed consent was obtained from all patients after a complete explanation of the procedures to be performed, and approval for this study was obtained from the Institutional Review Board of the Graduate School of Medicine, Chiba University, Japan.

DME patients with the central macular thickness $(\mathrm{CMT})>250 \mu \mathrm{m}$ were included in this study. Eyes with vitreomacular traction or epiretinal membrane were excluded. For STTA, $20 \mathrm{mg}$ of triamcinolone acetonide (TA) was injected into the posterior sub-Tenon space. The DMEs were classified by their OCT (SD-OCT, Heidelberg Engineering, Heidelberg, Germany) features: patients with SRD (SRD+;
38 eyes) and patients without SRD (non-SRD; 100 eyes). SRD was considered present if a nonreflective cavity with minimal shadowing of the underlying tissues exited under the posterior surface of the retina. ${ }^{25}$ In the non-SRD group, 61 eyes underwent STTA and the other 39 eyes underwent STTA + MAPC, while in the SRD+ group, 22 eyes underwent STTA, and the other 16 eyes underwent STTA + MAPC.

Twenty-six patients (18.8\%) underwent reinjection of STTA more than 3 months after the first injection. The indication for follow-up reinjection of STTA was an eye initially having a $\geq 30 \%$ decrease of the CMT compared with the baseline after first injection but then increasing by $\geq 30 \%$ or an eye having $<30 \%$ decrease of the CMT 3 months after first STTA injection.

The indications for MAPC were not specific, but the patients with MAs at the posterior pole underwent STTA combined with MAPC immediately or within 1 month after the STTA injection. Before MAPC, fluorescein angiography (FA) was performed and examined the leakage from MAs at the posterior pole. FA findings of active leaking MAs showed hyperfluorescent spot in early phase with increasing hyperfluorescence in the late phase. We performed focal MAPC in eyes with macular edema with CMT $>250 \mu \mathrm{m}$ accompanied with FA-guided active leaking MAs outside the foveal avascular zone. MAPC was performed with the following parameters: power $=80-100 \mathrm{~mW}$, duration $=100$ $\mathrm{ms}$, spot size $=100 \mu \mathrm{m}$. When new MA formation was observed after MAPC, we performed additional MAPC, but the minimal interval of retreatment was a month. This is not as per ETDRS guideline, but because of focal MAPC, we did not keep 3 months interval for retreatment of MAPC.

The baseline clinical data and characteristics of all of the patients are listed in Table 1. All characteristics including age, sex, level of glycosylated hemoglobin $\left(\mathrm{HbA}_{1 \mathrm{c}}\right)$, types of diabetic mellitus, insulin use, oral treatment, history of proteinurea, best-corrected visual acuity (BCVA) before treatment, and CMT before treatment were not significantly different among the four groups (Table 1).

The BCVA was measured before and 1, 3, and 6 months after the procedures. The CMT was measured by spectral-domain OCT at the same times. The data are presented as the mean \pm standard deviation. Statistical analyses were performed by paired $t$-tests, one-way analysis of variance (ANOVA), and repeated measures ANOVA. A $P<0.05$ was considered significant.

\section{Results}

Changes of the BCVA and the CMT as a function of postoperative time in eyes with DME are displayed in Table 2 and Figure 1. 
Table I Clinical data and features

\begin{tabular}{|c|c|c|c|c|c|}
\hline & \multicolumn{2}{|c|}{ Treatment: STTA injection } & \multicolumn{2}{|c|}{$\begin{array}{l}\text { Treatment: STTA injection } \\
\text { combined with MAPC }\end{array}$} & \multirow[t]{2}{*}{$P$-value } \\
\hline & SRD+ & Non-SRD & SRD+ & Non-SRD & \\
\hline Number of patients & 61 & 22 & 39 & 16 & \\
\hline Age (years) & $60.7 \pm 13.0$ & $60.8 \pm 11.7$ & $60.3 \pm 12.1$ & $60.4 \pm 15.3$ & 0.9988 \\
\hline $\mathrm{HbA}_{\mathrm{Ic}}(\%)$ & $6.7 \pm 1.1$ & $7.0 \pm 1.0$ & $6.4 \pm 1.1$ & $7.5 \pm 1.9$ & 0.176 \\
\hline Sex (male:female) & $31: 30$ & $16: 6$ & $22: 17$ & $10: 6$ & 0.3434 \\
\hline DM type (I:2) (\%) & $8: 92$ & $5: 95$ & $0: 100$ & $6: 94$ & 0.3429 \\
\hline Insulin use (\%) & 31 & 23 & 31 & 25 & 0.8991 \\
\hline Medication (\%) & 49 & 41 & 49 & 50 & 0.9195 \\
\hline Proteinuria (\%) & 31 & 50 & 38 & 31 & 0.4810 \\
\hline logMAR VA (before treatment) & $0.56 \pm 0.36$ & $0.60 \pm 0.44$ & $0.61 \pm 0.33$ & $0.5 \mathrm{I} \pm 0.29$ & 0.7636 \\
\hline CMT $(\mu \mathrm{m})$ (before treatment) & $545 \pm 147$ & $544 \pm 110$ & $512 \pm 202$ & $590 \pm 184$ & 0.3798 \\
\hline
\end{tabular}

Notes: Data are expressed as mean \pm standard deviation, unless otherwise specified.

Abbreviations: CMT, central macular thickness; DM, diabetes mellitus; HbA ${ }_{1 \mathrm{c}}$, glycosylated hemoglobin; MAPC, microaneurysm photocoagulation; non-SRD, patients without SRD; SRD, serous retinal detachment; SRD+, patients with SRD; STTA, sub-Tenon's capsule triamcinolone acetonide; VA, visual acuity.

The mean BCVA was not significantly improved at 6 months after treatment in both the non-SRD group and the SRD+ group after STTA $(P=0.0857$ and $P=0.7784$; paired $t$-tests). The mean BCVA was also not significantly improved in the SRD+ group after STTA + MAPC at 6 months after treatment ( $P=0.6282$; paired $t$-tests), but it was significantly improved in the non-SRD group after STTA + MAPC at 6 months after treatment ( $P=0.0473$; paired $t$-tests). The differences in the BCVAs among the four groups were not significant ( $P=0.2972$ by repeated measures ANOVA; Figure 1A).

The findings in a representative case in the STTA + MAPC non-SRD group are shown in Figure 2.

The CMTs in all four groups were significantly improved 6 months after treatment (STTA non-SRD, $P<0.0001$; STTA + MAPC non-SRD, $P<0.0001$; STTA SRD,$+ P=0.0064$; STTA + MAPC, $P<0.0001$; all paired $t$-tests). In the STTA + MAPC non-SRD group, the CMT was reduced significantly more than in the STTA + MAPC SRD+ group ( $P=0.0159$, repeated measures ANOVA; Table 2 and Figure 1B).

Twenty-eight patients (20\%) had temporary elevated intraocular pressure (IOP) $(>21 \mathrm{mmHg})$, which reduced with antiglaucoma eye drops. One patient $(0.7 \%)$ developed cataract formation but underwent cataract surgery successfully. No endophthalmitis was observed during the follow-up period.

\section{Discussion}

The results indicate that STTA is more effective in the SRDgroup than in the SRD+ group and that STTA combined with MAPC can improve the BCVA in the non-SRD group of patients with DME. However, some precaution in interpreting these results is necessary because this was a retrospective study and the follow-up period was short. Future prospective

Table 2 Changes of the BCVA and the CMT before and after treatment

\begin{tabular}{|c|c|c|c|c|}
\hline & \multicolumn{2}{|c|}{ Treatment: STTA injection } & \multicolumn{2}{|c|}{$\begin{array}{l}\text { Treatment: STTA injection combined } \\
\text { with MAPC }\end{array}$} \\
\hline & SRD+ & Non-SRD & SRD+ & Non-SRD \\
\hline Number of patients & 61 & 22 & 39 & 16 \\
\hline logMAR VA (before treatment) & $0.56 \pm 0.36$ & $0.60 \pm 0.44$ & $0.6 I \pm 0.33$ & $0.51 \pm 0.29$ \\
\hline logMAR VA at I month & $0.49 \pm 0.29$ & $0.53 \pm 0.38$ & $0.53 \pm 0.36$ & $0.53 \pm 0.32$ \\
\hline logMAR VA at 3 months & $0.46 \pm 0.33$ & $0.68 \pm 0.47$ & $0.52 \pm 0.31$ & $0.56 \pm 0.28$ \\
\hline logMAR VA at 6 months & $0.5 \pm 0.38$ & $0.64 \pm 0.5$ & $0.54 \pm 0.36 *$ & $0.5 I \pm 0.25$ \\
\hline CMT $(\mu \mathrm{m})$ (before treatment) & $545 \pm 147$ & $544 \pm 110$ & $512 \pm 202$ & $590 \pm 184$ \\
\hline CMT at I month & $366 \pm 118$ & $407 \pm 124$ & $360 \pm 172$ & $435 \pm 179$ \\
\hline CMT at 3 months & $354 \pm|3|$ & $390 \pm 123$ & $34 I \pm 123$ & $460 \pm 159$ \\
\hline CMT at 6 months & $401 \pm 166^{* *}$ & $408 \pm 150 * *$ & $373 \pm 183 * *, * * *$ & $509 \pm 170 * *$ \\
\hline
\end{tabular}

Notes: Data are expressed as mean \pm standard deviation. $* P<0.05$ (paired $t$-test); before treatment versus 6 months after treatment; $* * P<0.01$ (paired $t$-test); before treatment versus 6 months after treatment; ***P $<0.05$ (repeated measured ANOVA); STTA injection combined with MAPC in patients without SRD versus STTA injection combined with MAPC in patients with SRD.

Abbreviations: ANOVA, one-way analysis of variance; BCVA, best-corrected visual acuity; CMT, central macular thickness; MAPC, microaneurysm photocoagulation; nonSRD, patients without SRD; SRD, serous retinal detachment; SRD+, patients with SRD; STTA, sub-Tenon's capsule triamcinolone acetonide; VA, visual acuity. 


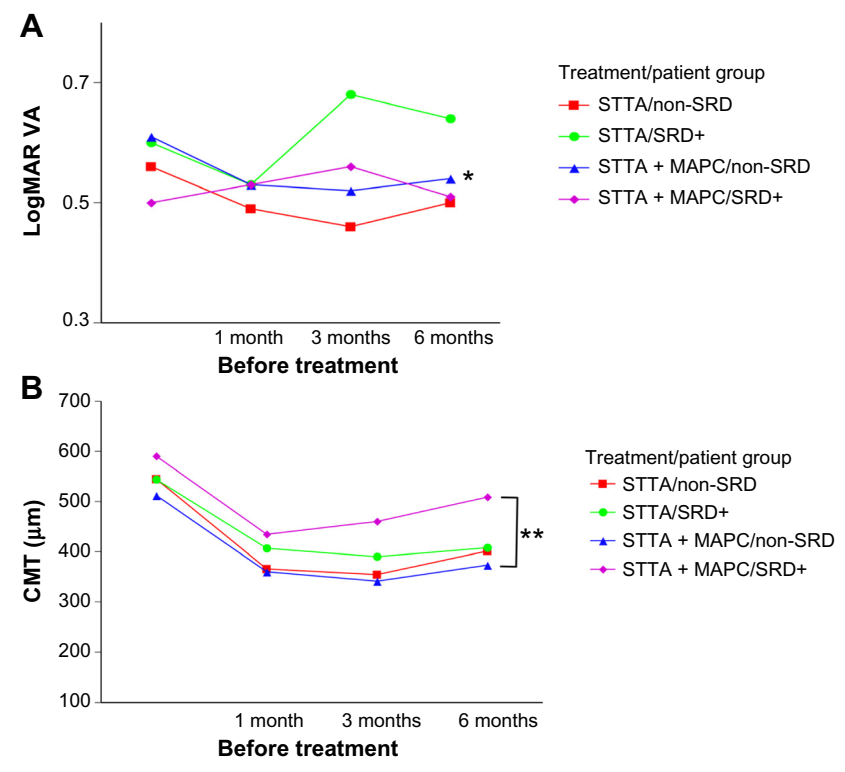

Figure I Changes of the BCVA and the CMT as a function of postoperative time in eyes with DME.

Notes: (A) The BCVA was significantly improved in the non-SRD group at 6 months after STTA + MAPC; $* P<0.05$ (paired $t$-test). (B) Although the CMTs of all groups were significantly reduced 6 months after treatment, the CMT of the nonSRD group after STTA + MAPC was reduced significantly more in the SRD+ group after STTA + MAPC; **P $<0.05$ (repeated measures ANOVA).

Abbreviations: ANOVA, one-way analysis of variance; BCVA, best-corrected visual acuity; CMT, central macular thickness; DME, diabetic macular edema; MAPC, microaneurysm photocoagulation; non-SRD, patients without SRD; SRD, serous retinal detachment; SRD+, patients with SRD; STTA, sub-Tenon triamcinolone acetonide; STTA + MAPC, STTA injection combined with MAPC; VA, visual acuity.

studies with longer follow-up periods are needed to confirm the effects of STTA on the SRD in eyes with DME, and also the additive effects of MAPC on DME.

One of the major complications of TA is a cataract formation. However, in this study, only one eye required cataract surgery after treatment. In the previous study, $83 \%$ of eyes required cataract surgeries after 4\% IVTA injection during 3-year follow-up period. ${ }^{26}$ In case of IVTA, triamcinolone can directly affect lens because the triamcinolone is injected into the intraocular space. However, in STTA, triamcinolone is injected into the posterior sub-Tenon space. Thus, the adverse effect of triamcinolone on lens seems to be indirect and milder after STTA than after IVTA.

MA formation in the posterior pole may be an initial and visible sign of increasing diabetic stress in the macula area of patients with DME. In fact, the results of recent studies indicated that a high MA turnover rate, ie, sum of MA formation and disappearance, is a high risk factor for the development of clinically significant macular edema. In addition, MA formation rates may be a predictive marker for the progression of macular edema. ${ }^{13,14}$

In the SRD+ group, the effect of STTA combined with MAPC appeared to be worse than that of STTA alone.
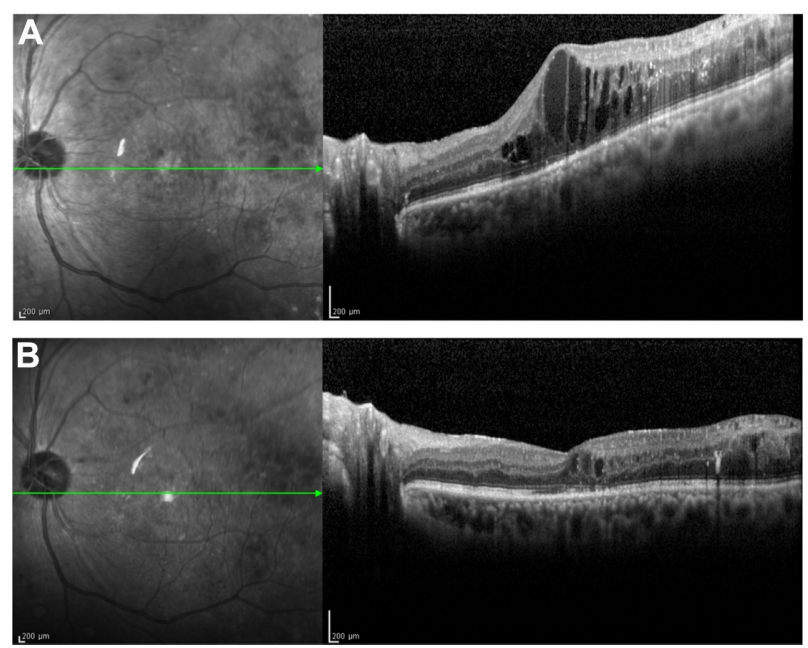

Figure 2 Representative case of DME treated with STTA injection combined with MAPC.

Notes: A 57-year-old man underwent pars plana vitrectomy for proliferative diabetic retinopathy accompanied by vitreous hemorrhage. (A) Three months later, DME has developed. The BCVA is 0.4 in the left eye. The patient was then treated with STTA injection combined with MAPC. (B) Three months later, the DME improved. The final BCVA was 0.6 in the left eye. No recurrence has been observed during the follow-up period.

Abbreviations: DME, diabetic macular edema; STTA, sub-Tenon triamcinolone acetonide; MAPC, microaneurysm photocoagulation; BCVA, best-corrected visual acuity.

This indicated that the retinas in the SRD+ group with MAs may be under more severe diabetic stress than the non-SRD group.

STTA was not more effective on the SRDs in eyes with DME than in eyes with no SRD with DME. MA formation may cause an increase of vascular permeability, and the leaked components may be pooled in the subretinal space causing the SRD. However, STTA combined with MAPC did not show any additive effects on these eyes. Thus, the leakage from MA may not be the main cause for the development of the SRD in these patients. The results of several earlier studies indicated that a dysfunction of the retinal pigment epithelium (RPE) is present in diabetic retinas. ${ }^{27,28}$ Thus, the dysfunctional RPE of diabetic retinas may not be able to remove the excess fluid in the subretinal space. Because steroids may affect the function of the RPE, ${ }^{29}$ STTA alone may not be sufficient to improve the SRD in eyes with DME. Because the intravitreal injection of anti-VEGF drugs is also less effective on SRD+ than non-SRD, ${ }^{30}$ other combination therapies or vitrectomy may be better than STTA for SRD in eyes with DME.

Our results indicate that STTA combined with MAPC in the non-SRD group can improve the BCVA only 6 months after treatment. The results of an earlier study indicated that IVTA combined with grid laser photocoagulation was more effective for diffuse DME than IVTA only. ${ }^{31}$ Shimura et al suggest that pretreatment of STTA has beneficial effects for grid laser in diffuse DME. ${ }^{31}$ Steroids increase the effectiveness of 
laser photocoagulation with lower power because they reduce retinal thickness. In addition, steroids may promote more mature laser scar formation. ${ }^{12}$ MAPC reduces leakage from the MAs by closure of the MAs. ${ }^{15}$ Although MAPC is not for all patients with DME, STTA combined with MAPC may be considered if MAs are found in the posterior pole.

\section{Conclusion}

Our findings indicate that STTA is more effective in the non-SRD type of DME eyes than in the SRD+ type of eyes with DME, and that MAPC may have an additive effect in the non-SRD type of eyes with DME. Thus, the type of DME should be determined before STTA or combined with MAPC is used to treat eyes with DME.

\section{Acknowledgments}

This study was supported by a grant from the Charitable Trust Fund for Ophthalmic Research in Commemoration of Santen Pharmaceutical's Founder and a grant-in aid from the Ministry of Education, Science, Sports, and Culture of the Japanese Government. The authors thank Professor Duco Hamasaki for editing the manuscript.

\section{Author contributions}

TO and SN conducted the data analysis and wrote the manuscript. All authors performed the STTA and/or MAPC and were involved with the data collection. TO and SY designed, wrote, and edited the manuscript. All authors contributed toward data analysis, drafting, and revising the paper, gave final approval of the version to be published, and agree to be accountable for all aspects of the work.

\section{Disclosure}

The authors report no conflicts of interest in this work.

\section{References}

1. O'Doherty M, Dooley I, Hickey-Dwyer M. Interventions for diabetic macular oedema: a systematic review of the literature. Br J Ophthalmol. 2008;92(12):1581-1590.

2. Early Treatment Diabetic Retinopathy Study Research Group. Photocoagulation for diabetic macular edema: Early Treatment Diabetic Retinopathy Study report number 1. Arch Ophthalmol. 1985;103(12):1796-1806.

3. Lovestam-Adrian M, Agardh E. Photocoagulation of diabetic macular oedema-complications and visual outcome. Arch Ophthalmol Scand. 2000;78(6):667-671.

4. Ohguro N, Okada AA, Tano Y. Trans-Tenon's retrobulbar triamcinolone infusion for diffuse diabetic macular edema. Graefes Arch Clin Exp Ophthalmol. 2004;242(5):444-445.

5. Nonomura S, Oshitari T, Arai M, et al. The effect of posterior sub-Tenon's capsule triamcinolone acetonide injection to that of pars plana vitrectomy for diabetic macular edema. Clin Ophthalmol. 2014;8:825-830.

6. Ho TC, Lai WW. Intravitreal triamcinolone acetonide for diabetic macular edema. Ophthalmologica. 2006;220(5):349-350.
7. Mitchell P, Bandello F, Schmidt-Erfurth U, et al. The RESTORE study: ranibizumab monotherapy or combined with laser versus laser monotherapy for diabetic macular edema. Ophthalmology. 2011;118(4): 615-625.

8. Diabetic Retinopathy Clinical Research Network, Elman MJ, Qin H, Aiello LP, et al. Intravitreal ranibizumab for diabetic macular edema with prompt versus deferred laser treatment: three-year randomized trial results. Ophthalmology. 2012;119(11):2312-2318.

9. Sultan MB, Zhou D, Loftus J, Dombi T, Ice KS, Macugen 1013 Study Group. A phase 2/3, multicenter, randomized, double-masked, 2-year trial of pegaptanib sodium for the treatment of diabetic macular edema. Ophthalmology. 2011;118(6):1107-1118.

10. Do DV, Nguyen QD, Boyer D, et al. One-year outcomes of the da Vinci Study of VEGF Trap-Eye in eyes with diabetic macular edema. Ophthalmology. 2012;119(8):1658-1665.

11. Takatsuna Y, Yamamoto S, Nakamura Y, Tatsumi T, Arai M, Mitamura Y. Long-term therapeutic efficacy of the subthreshold micropulse diode laser photocoagulation for diabetic macular edema. Jpn J Ophthalmol. 2011;55(4):365-369.

12. Zur D, Loewenstein A. Combined therapy for diabetic macular edema. J Ophthalmol. 2012;2012:ID484612.

13. Ribeiro ML, Nunes SG, Cunha-Vaz JG. Microaneurysm turnover at the macula predicts risk of development of clinically significant macular edema in persons with mild nonproliferative diabetic retinopathy. Diabetes Care. 2013;36(5):1254-1259.

14. Haritoglou C, Kernt M, Neubauer A, et al. Microaneurysm formation rate as a predictive marker for progression to clinically significant macular edema in nonproliferative diabetic retinopathy. Retina. 2014; 34(1):157-164.

15. Lee SN, Chhablani J, Chan CK, et al. Characterization of microaneurysm closure after focal laser photocoagulation in diabetic macular edema. Am J Ophthalmol. 2013;155(5):905-912.

16. Kern TS. Contributions of inflammatory processes to the development of the early stages of diabetic retinopathy. Exp Diabetes Res. 2007; 2007:ID95103.

17. Nauck M, Roth M, Tamm M, et al. Induction of vascular endothelial growth factor by platelet-activating factor and platelet-derived growth factor is downregulated by corticosteroids. Am J Respir Cell Mol Biol. 1997;16(4):398-406.

18. Felinski EA, Antonetti DA. Glucocorticoid regulation of endothelial cell tight junction gene expression: novel treatments for diabetic retinopathy. Curr Eye Res. 2005;30(11):949-957.

19. Sakamoto T, Hida T, Tano Y, et al. Committee for Triamcinolone Acetonide for Ocular Disease in Japan: survey of triamcinolone acetonide for ocular diseases in Japan. Nihon Ganka Gakkai Zasshi. 2007;111(12):936-945.

20. Otani T, Kishi S, Maruyama Y. Patterns of diabetic macular edema with optical coherence tomography. Am J Ophthalmol. 1999;127(6):688-693.

21. Ozdemir H, Karacorlu M, Karacorlu SA. Regression of serous macular detachment after intravitreal triamcinolone acetonide in patients with diabetic macular edema. Am J Ophthalmol. 2005;140(2): 251-255.

22. Shukla D, Behera UC, Chakraborty S, Mahalakshmi R, Prasad NM. Serous macular detachment as a predictor of resolution of macular edema with intravitreal triamcinolone injection. Ophthalmic Surg Lasers Imaging. 2009;40(2):115-119.

23. Shimura M, Yasuda K, Nakazawa T, et al. Visual outcome after intravitreal triamcinolone acetonide depends on optical coherence tomographic patterns in patients with diffuse diabetic macular edema. Retina. 2011;31(4):748-754.

24. Kawashima H, Mizukawa K, Kiryu J. Factors associated with visual recovery after sub-Tenon injection of triamcinolone acetonide in diabetic macular edema. Clin Ophthalmol. 2012;6:1307-1314.

25. Ozdemir H, Karacorlu M, Karacorlu SA. Regression of serous macular detachment after intravitreal triamcinolone acetonide in patients with diabetic macular edema. Am J Ophthalmol. 2005;140(2):251-255 
26. Diabetic Retinopathy Clinical Research Network (DRCR.net), Beck RW, Edwards AR, Aiello LP, et al. Three-year follow-up of a randomized trial comparing focal/grid photocoagulation and intravitreal triamcinolone for diabetic macular edema. Arch Ophthalmol. 2009; 127(3):245-251.

27. Decanini A, Karunadharma PR, Nordgaard CL, Feng X, Olsen TW, Ferrington DA. Human retinal pigment epithelium proteome changes in early diabetes. Diabetologia. 2008;51(6):1051-1061.

28. Omri S, Behar-Cohen F, de Kozak Y, et al. Microglia/macrophages migrate through retinal epithelium barrier by a transcellular route in diabetic retinopathy: role of $\mathrm{PKC} \zeta$ in the Goto Kakizaki rat model. Am J Pathol. 2011;179(2):942-953.
29. Valamanesh F, Torriglia A, Savoldelli M, et al. Glucocorticoids induce retinal toxicity through mechanisms mainly associated with paraptosis. Mol Vis. 2007;13:1746-1757.

30. Shimura M, Yasuda K, Yasuda M, Nakazawa T. Visual outcome after intravitreal bevacizumab depends on the optical coherence tomographic patterns of patients with diffuse diabetic macular edema. Retina. 2013;33(4):740-747.

31. Shimura M, Nakazawa T, Yasuda K, Shiono T, Nishida K. Pretreatment of posterior subtenon injection of triamcinolone acetonide has beneficial effects for grid pattern photocoagulation against diffuse diabetic macular oedema. Br J Ophthalmol. 2007;91(4):449-454.

\section{Publish your work in this journal}

The International Medical Case Reports Journal is an international, peer-reviewed open-access journal publishing original case reports from all medical specialties. Previously unpublished medical posters are also accepted relating to any area of clinical or preclinical science. Submissions should not normally exceed 2,000 words or
4 published pages including figures, diagrams and references. The manuscript management system is completely online and includes a very quick and fair peer-review system, which is all easy to use. Visit http://www.dovepress.com/testimonials.php to read real quotes from published authors.

Submit your manuscript here: http://www.dovepress.com/international-medical-case-reports-journal-journal 